\title{
Manejo da fratura supracondiliana do úmero na criança
}

\section{Management of Supracondylar Humeral Fracture in Children}

\author{
Pedro Poggiali ${ }^{1}$ Francisco Carlos Salles Nogueira ${ }^{10}$ Maria Paula de Mello Nogueira1® \\ ${ }^{1}$ Pediatric Orthopedic Surgery, Rede Mater Dei de Saúde, Belo \\ Horizonte, Minas Gerais, Brazil \\ Endereço para correspondência Pedro Poggiali, MD, Rua Araguari, \\ 1156/1901, Clínica Poggiali, Belo Horizonte, MG, 30190-111, Brazil \\ (e-mail: pedro@poggiali.com.br).
}

Rev Bras Ortop 2022;57(1):23-32.

\section{Resumo \\ Palavras-chave \\ - fraturas do úmero \\ - cotovelo \\ - criança \\ - fixação de fratura}

A fratura supracondiliana do úmero representa cerca de 3 a 15\% de todas as fraturas na criança, sendo a que mais requer tratamento cirúrgico na população pediátrica. Apesar de os avanços no tratamento e na assistência terem contribuído para uma redução drástica da complicação mais temida, a contratura isquêmica de Volkmann, os riscos inerentes à fratura permanecem. Ausência de pulso palpável em fraturas tipo III é reportada em até $20 \%$ dos casos. Uma cuidadosa avaliação sensitiva, motora e vascular do membro acometido é fundamental na determinação da urgência do tratamento. Crianças mais velhas, sexo masculino, cotovelo flutuante, e lesão neurovascular são fatores de risco para a síndrome de compartimento. A cominuição medial pode levar à consolidação em varo, mesmo nos casos aparentemente inocentes. O método de escolha para o tratamento da fratura desviada é a redução fechada e fixação percutânea. Os erros na fixação e posicionamento inadequado dos implantes são as principais causas de perda de redução. Já existem evidências suficientes para a utilização de um terceiro fio de Kirschner, lateral ou medial, nas fraturas instáveis (tipo III e IV). Baseado nos conceitos atuais, um fluxograma para o tratamento da fratura supracondiliana do úmero na criança é sugerido pelos autores.

Supracondylar humeral fracture represents $\sim 3$ to $15 \%$ of all fractures in children. It is the fracture that most requires surgical treatment in the pediatric population. Advances in treatment and care have contributed to a reduction in the most dramatic complication: Volkmann ischemic contracture. Nevertheless, the risks inherent to the fracture remain. Absence of palpable pulse in type-III fractures is reported in up to $20 \%$ of the cases. Careful sensory, motor, and vascular evaluation of the affected limb is crucial in determining the urgency of treatment. Older children, male patients, floating elbow, and neurovascular injury are risk factors for compartment syndrome. Medial comminution can lead to varus malunion, even in apparently innocent cases. The recommended treatment of displaced fractures is closed reduction and percutaneous pinning. Technical errors in pin placement are the main cause of loss of reduction. There is enough evidence for the addition of a third lateral or medial Kirschner wire in unstable fractures (types III and IV). Medial comminution may lead to cubitus varus even in mild displaced fractures. Based on current concepts, a flowchart for the treatment of supracondylar humeral fracture in children is suggested by the authors. recebido

17 de Agosto de 2019

aceito

27 de Janeiro de 2020

Publicado on-line

Julho 23, 2020
DOI https://doi.org/

10.1055/s-0040-1709734. ISSN 0102-3616. (c) 2020. Sociedade Brasileira de Ortopedia e Traumatologia. All rights reserved.

This is an open access article published by Thieme under the terms of the Creative Commons Attribution-NonDerivative-NonCommercial-License, permitting copying and reproduction so long as the original work is given appropriate credit. Contents may not be used for commercial purposes, or adapted, remixed, transformed or built upon. (https://creativecommons.org/ licenses/by-nc-nd/4.0/)

Thieme Revinter Publicações Ltda., Rua do Matoso 170, Rio de Janeiro, RJ, CEP 20270-135, Brazil 


\section{Introdução}

Entre as lesões traumáticas do esqueleto imaturo, a fratura supracondiliana do úmero (FSU) destaca-se não só por sua elevada frequência, mas também pelos riscos que a acompanham. Os avanços no tratamento e na assistência contribuíram para melhores resultados e para a redução drástica da complicação mais temida: a contratura isquêmica de Volkmann. ${ }^{1-4}$ Mas os riscos inerentes à fratura permanecem, assim como a justificada apreensão dos cirurgiões que rotineiramente lidam com essa lesão.

Mais comum em pacientes entre 3 e 10 anos de idade, a FSU tem seu pico de incidência aos 6 anos de idade., ${ }^{1,4}$ Representa cerca de 3 a 15\% de todas as fraturas em crianças, chegando a $70 \%$ entre as do cotovelo. Com incidência estimada de 1,7 para cada 1.000 indivíduos, é a fratura que mais requer tratamento cirúrgico na população pediátrica. ${ }^{4,8-12}$

O mecanismo de trauma mais frequente é a queda com a mão espalmada gerando a hiperextensão do cotovelo. Com isso, o olécrano age como um fulcro posterior no úmero, resultando na fratura tipo extensão, responsável por 97 a $98 \%$ dos casos. ${ }^{1,4}$ A fratura tipo flexão, mais rara, é causada por trauma posterior no cotovelo fletido, resultando no deslocamento anterior do fragmento distal. ${ }^{4}$

\section{Classificação}

Gartland dividiu as fraturas em três tipos: sem desvio, desvio moderado, e desvio extremo. ${ }^{3,13}$ Posteriormente, Wilkins modificou a classificação ao incluir o conceito do contato cortical posterior. ${ }^{3,14}$ É a classificação mais utilizada, com alta concordância intraobservador e interobservador ( - Figura 1).,

Tipo I: sem desvio, ou minimamente desviada ( $<2 \mathrm{~mm})$. 0 periósteo íntegro em toda a circunferência mantém a estabilidade. ${ }^{3}$ A linha de fratura pode não ser visível na radiografia inicial, com o sinal do coxim gorduroso sendo a única evidência da lesão. ${ }^{4}$ Nesse caso, a reação periosteal, que normalmente surge após a segunda semana, confirma a suspeita clínica.
Tipo II: fratura desviada, mantendo contato cortical posterior com uma dobradiça preservada. ${ }^{3} \mathrm{Na}$ radiografia em perfil, a linha umeral anterior não corta o terço médio do capitelo. É o tipo de fratura com maior discordância entre os autores, sendo que alguns consideram que qualquer desvio no plano coronal seria suficiente para classificá-la como tipo III. ${ }^{1,4}$ Porém a integridade da dobradiça cortical posterior mantém certa estabilidade mesmo na presença de alguma rotação ou cominuição. A alternativa sugerida por Wilkins é a subdivisão em II A (desvio apenas em extensão) e II B (radiografia em ânteroposterior [AP] evidenciando desvio rotacional ou angular, porém com contato cortical posterior preservado na radiografia em perfil). ${ }^{3}$ A diferenciação em subtipos II A e II B é válida por ajudar a identificar fraturas estáveis com desvio apenas em extensão e que poderiam ser submetidas a uma tentativa inicial de tratamento não cirúrgico. Fraturas com desvio rotacional ou angular tendem a ser mais instáveis e propensas à perda de redução quando não fixadas (-Figura 2). ${ }^{7,15-17}$ Uma potencial armadilha é subestimar a fratura com pouco desvio em extensão, mas com cominuição da coluna medial, pois seu colapso pode levar à consolidação em varo, mesmo nos casos aparentemente inocentes. ${ }^{1,4}$

Tipo III: desvio completo, sem contato entre as corticais, com maior risco de lesões neurovasculares e interposição de partes moles. ${ }^{3,11}$ São fraturas instáveis e geralmente de difícil redução. Porém, o periósteo posterior parcialmente preservado ajuda na redução e estabilização da fratura quando o cotovelo é fletido, facilitando a fixação. ${ }^{4}$

Tipo IV: instabilidade multidirecional, o periósteo rompido em toda sua circunferência torna a fratura extremamente instável. ${ }^{1,18}$ Descrita por Leitch et al., ${ }^{18}$ essa lesão pode ser confirmada durante a tentativa de redução sob fluoroscopia, quando a fratura se mostra instável, tanto em flexão quanto em extensão. 3,19

\section{Tratamento}

O tratamento da fratura tipo I é não cirúrgico: imobilização do cotovelo com tala axilopalmar posterior em flexão de 60 a $80^{\circ}$ por 3 semanas. ${ }^{1,9} \mathrm{O}$ controle radiográfico em torno de 7

\section{Classificação}

\begin{tabular}{|l|l|}
\hline Tipo I & Sem desvio ou mínimo desvio \\
\hline Tipo II & $\begin{array}{l}\text { Desvio em extensão, mantendo contato cortical com dobradiça posterior preservada } \\
\text { II-A: sem desvio no plano coronal } \\
\text { II-B: rotação ou angulação no plano coronal. }\end{array}$ \\
\hline Tipo III & Sem contato entre as corticais. \\
\hline Tipo IV & $\begin{array}{l}\text { Instabilidade multidirecional devido ruptura circunferencial do periósteo, a instabilidade } \\
\text { tanto em flexão quanto em extensão pode ser confirmada sob fluoroscopia }\end{array}$ \\
\hline
\end{tabular}

Fig. 1 Classificação de Gartland modificada, incluindo fraturas dos tipos IV e II B. 

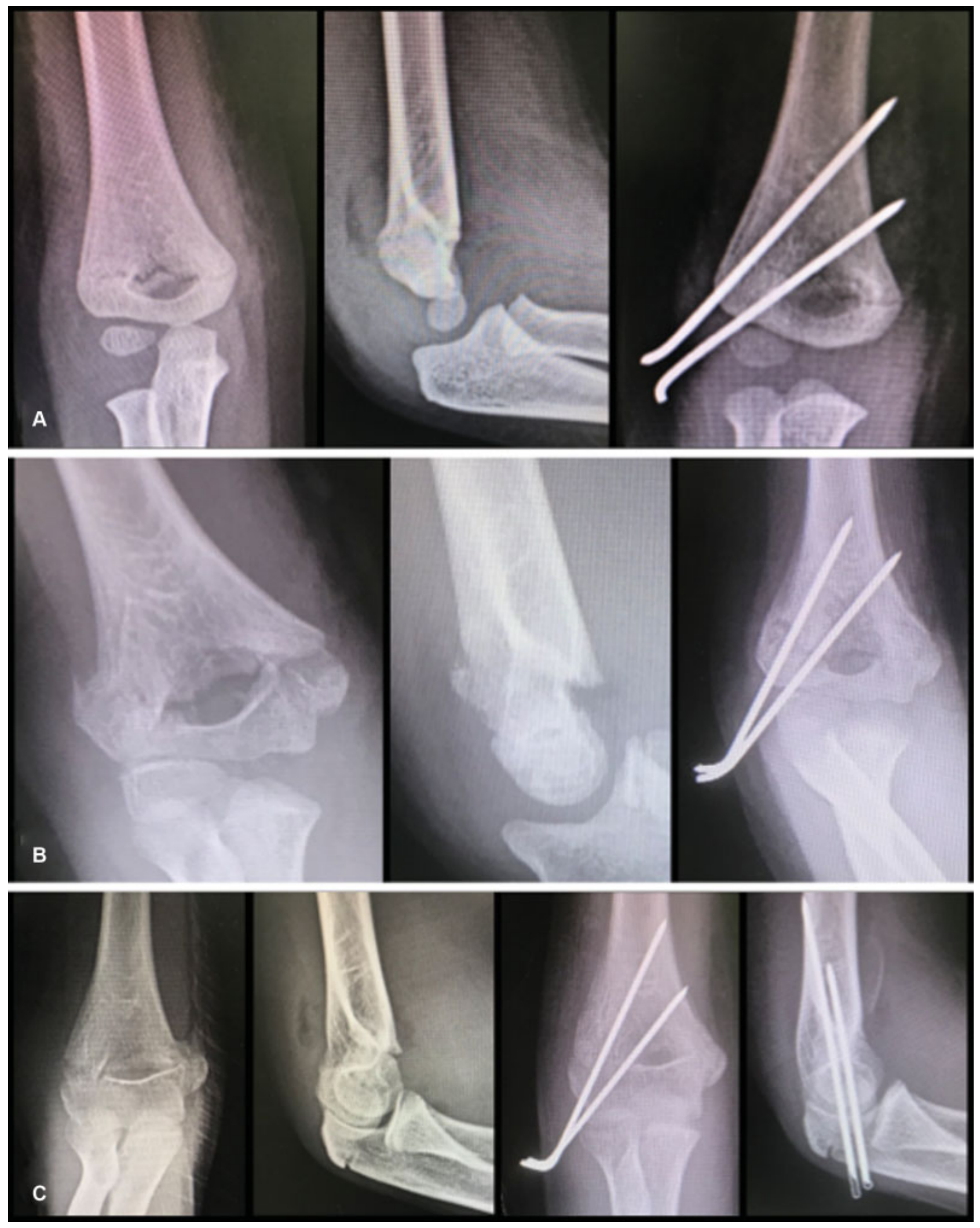

Fig. 2 Exemplos de fraturas tipo II. (A) Fratura tipo II A, desvio apenas em extensão. (B) Fratura com desvio em extensão, rotação e angulação, tipo II B. (C) Fratura com cominuição medial, tipo II B.

dias é fundamental para a detecção precoce de eventual desvio. 4

Alguns estudos sugerem que fraturas estáveis com desvio apenas em extensão (tipo II A) podem ser tratadas inicia-
Imente com redução fechada, imobilização e acompanhamento rigoroso para identificar perda de redução. ${ }^{15-17}$ Porém, conforme a diretriz da American Academy of Orthopaedic Surgeons, o método de escolha para o tratamento da 
FSU desviada é redução fechada e fixação percutânea. 9,10,20 Fratura na qual a linha umeral anterior não toca o capitelo, ou qualquer fratura com translação, rotação ou angulação coronal, deve ser reduzida e fixada. ${ }^{1,4,7}$

O exame físico ajuda na determinação da urgência do tratamento cirúrgico. ${ }^{11,21,22}$ É fundamental que seja feita uma cuidadosa avaliação sensitiva, motora e vascular do membro acometido. ${ }^{1,4,7}$ Edema acentuado, presença de equimose volar, e tensionamento da pele pelo fragmento proximal da fratura na região cubital são sinais de gravidade. Essas características indicam maior lesão de partes moles e risco elevado de lesão neurovascular associada ( Figura 3). 4,11

0 membro deve ser imobilizado com tala gessada bem acolchoada em flexão entre 30 a $40^{\circ}$ até que o paciente possa ser submetido a tratamento definitivo. ${ }^{1,4,7}$ Em casos extremos, com desvio grosseiro ou sem pulso palpável, a redução parcial da fratura com uma manobra de flexão do cotovelo até $40^{\circ}$ e tração leve pode melhorar a perfusão e aliviar a tensão das partes moles. ${ }^{4,8}$ A tentativa forçada de redução no pronto-socorro com imobilização do cotovelo em flexão maior que $80^{\circ}$ é contraindicada devido ao risco de síndrome de compartimento. ${ }^{4}$ Em caso de desvio acentuado, o paciente deve ser mantido em observação no hospital até a abordagem cirúrgica. $^{7}$

Não há consenso sobre o limite de tempo que uma fratura fechada com pulso palpável poderia aguardar. ${ }^{9,23}$ Diversos estudos mostram que adiar o tratamento cirúrgico, por até 24 horas em algumas séries, não implica em maior incidência de complicações, necessidade de redução aberta ou resultados insatisfatórios. ${ }^{1,4,7,24-26}$ Porém esses estudos clínicos estão sujeitos ao viés de seleção, uma vez que os casos mais graves tendem a ser abordados precocemente. ${ }^{4,26}$ A decisão deve ser individualizada e o tratamento o mais precoce possível, com atenção especial para os sinais de gravidade e exame neurovascular. ${ }^{4,11,21-26}$

\section{Métodos de fixação}

A fixação pode ser ser feita com dois ou três pinos, laterais ou cruzados. ${ }^{1,20,27}$ Os pinos de entrada lateral devem ser divergentes, buscando espaçamento máximo no foco da fratura e
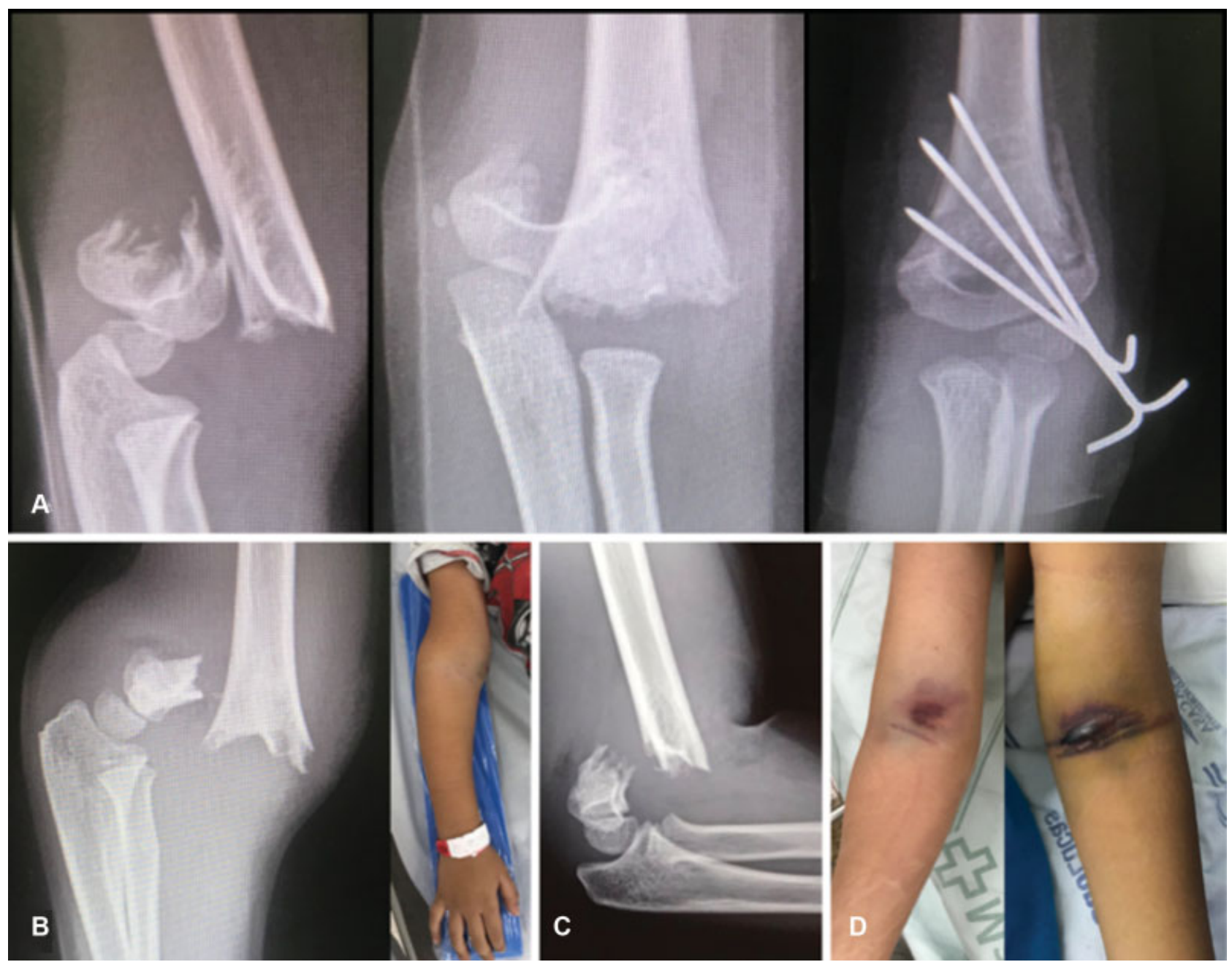

Fig. 3 Exemplos de fraturas tipo III e sinais de gravidade, indicando maior lesão de partes moles e risco elevado de lesões neurovasculares associadas. (A) Fratura tipo III fixada com 3 pinos laterais divergentes. (B) Fratura com desvio importante, edema acentuado e deformidade grosseira. (C) O fragmento proximal atravessa o músculo braquial e a fáscia anterior, tensionando a pele e criando uma prega volar no cotovelo que pode ser observada no exame clínico (pucker sign - sinal da prega). (D) sinal de Kirmisson, equimose volar transversa na prega de flexão do cotovelo. 
fixação tanto da coluna lateral quanto da medial. ${ }^{4,28}$ Podem ser paralelos, mas nunca convergentes, não devem cruzar no foco da fratura, e a fixação por pelo menos dois pinos bicorticais (nos fragmentos proximal e distal) é fundamental. ${ }^{1,6,7,27}$ De forma geral, dois fios de Kirschner laterais são suficientes para fratura tipo II; no entanto, já existem evidências suficientes para indicação de três fios para fraturas tipo III. ${ }^{4,6,27,29-32}$ A adição de um terceiro fio de Kirschner está relacionada ao menor risco de falha da fixação e à necessidade de revisão cirúrgica. ${ }^{6,31,32}$ Fios de $2.0 \mathrm{~mm}$ garantem mais estabilidade e devem ser considerados em pacientes maiores. $4,7,30,33$

Apesar de alguns estudos biomecânicos sugerirem que a fixação cruzada pode ser mais estável do que apenas dois pinos laterais, estudos clínicos evidenciam que a fixação apenas por via lateral é suficiente na maioria dos casos e que o uso rotineiro do pino de entrada medial deve ser evitado devido ao risco de lesão iatrogênica do nervo ulnar. 1,7,20,25,34-40 Estima-se que a neuropraxia do ulnar ocorra em 1 a cada 28 pacientes, cerca de $4 \%$ dos casos, quando realizada fixação cruzada. ${ }^{4,20,41,42}$ No entanto, algumas fraturas mais instáveis, com traço oblíquo ou cominuídas, podem exigir um pino de entrada medial para alcançar estabilização adequada após a fixação com dois ou três pinos laterais. ${ }^{30-32,39,43,44}$ Nesse caso, alguns cuidados diminuem o risco de lesão: estender o cotovelo até pelo menos $80^{\circ}$ para relaxar o nervo ulnar, que pode subluxar anteriormente durante a flexão, ou realizar pequeno acesso para visualização direta do ponto de entrada medial.4,31,34,44

\section{Redução aberta}

A redução cruenta está indicada nos casos de fraturas irredutíveis, expostas, ou quando ocorre diminuição da perfusão após a redução. ${ }^{1,4} \mathrm{O}$ acesso anterior permite a liberação das estruturas interpostas, usualmente volares, com visualização direta da artéria braquial e do nervo mediano, sendo, portanto, o acesso o mais recomendado atualmente. ${ }^{4,7,45} \mathrm{O}$ acesso lateral também é descrito com
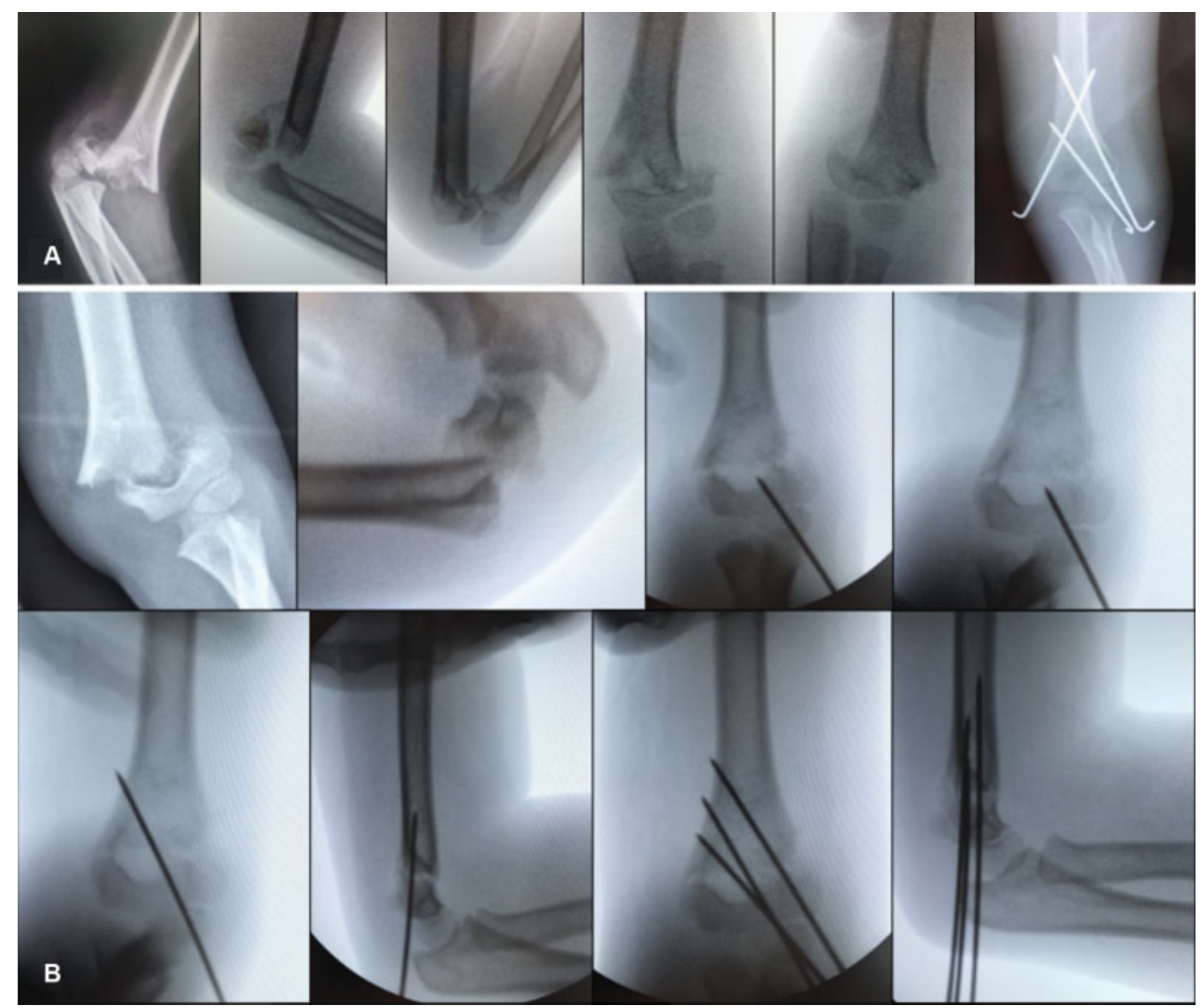

Fig. 4 (A) Fratura tipo IV, instabilidade multidirecional confirmada durante a tentativa de redução sob fluoroscopia. (B) Fratura tipo IV reduzida e fixada através da técnica de joystick. 
bons resultados. ${ }^{4}$ Já o acesso posterior apresenta as seguintes desvantagens: risco de necrose avascular da tróclea; aumento da instabilidade com a abertura do periósteo posterior; e maior incidência de rigidez. ${ }^{1,4}$

\section{Fratura tipo IV}

A fratura com instabilidade multidirecional oferece maior dificuldade de redução, mas não necessariamente exige abordagem cruenta. ${ }^{18,43}$ A técnica de joystick descrita por Novais et al. ${ }^{19}$ consiste na manipulação com inserção de um fio de Kirschner de $2.0 \mathrm{~mm}$ lateral apenas no fragmento distal, através do capitelo e apontando para o centro do foco de fratura. $\mathrm{O}$ arco do intensificador deve estar paralelo à maca para alternar entre as incidências AP e perfil, sem interferir na posição do braço. $\mathrm{O}$ assistente corrige a rotação do fragmento proximal até conseguir uma imagem em perfil verdadeiro do úmero e mantém essa posição durante todo o procedimento. A seguir, o cirurgião manipula o fragmento distal com auxílio do pino já inserido para corrigir a rotação, translação e angulação. Após obtido o alinhamento adequado, o pino é progredido até o fragmento proximal, e a fratura estabilizada com mais dois fios de Kirschner laterais divergindo do primeiro. Apesar da dificuldade técnica, tempo cirúrgico prolongado, maior incidência de redução aberta e maior necessidade de pino medial para estabilização adequada, resultados satisfatórios podem ser obtidos nas fraturas tipo IV (- Figura 4). ${ }^{18,19,43}$

\section{Fratura em flexão}

Apesar de a classificação de Gartland ter sido descrita para a fratura em extensão, ela também é aplicada para a fratura em flexão. ${ }^{20} \mathrm{O}$ tratamento segue a lógica descrita anteriormente: fratura sem desvio deve ser tratada de forma conservadora e fratura desviada deve ser reduzida e fixada. ${ }^{4}$ Alguns autores sugerem a tentativa de redução de fratura tipo II com a imobilização em extensão do cotovelo. ${ }^{14}$ Contudo, a tolerância para desvios deve ser baixa. 0 cirurgião deve estar atento à maior incidência de lesão nervosa e necessidade de redução aberta nessas fraturas. ${ }^{12,41,46,47}$ As fraturas irredutíveis podem ser abordadas por via anteromedial, medial, ou posterior, preservando o periósteo anterior íntegro e permitindo a visualização direta do nervo ulnar.,46

\section{Complicações}

\section{Perda de redução}

A perda de redução ocorre em torno de $4 \%$ dos casos, sendo sua principal causa a fixação inadequada. ${ }^{4,6,27,28}$ Pinos cruzando no foco, convergentes, ou sem espaçamento adequado deixam a osteossíntese instável. ${ }^{28}$ Outro erro potencial é não conseguir a fixação com pelo menos dois pinos bicorticais. Isso geralmente ocorre quando um dos pinos fica intramedular no fragmento proximal ou passando através do foco da fratura. ${ }^{6,27}$ Caso haja dúvida sobre o posicionamento adequado dos implantes ou da estabilidade da fixação, a inserção de um terceiro ou até um quarto pino aumenta a chance de sucesso. ${ }^{4,27,29-32} \mathrm{Um}$ fator a ser considerado é a redução inadequada: a rotação diminui o suporte das colunas lateral e medial no fragmento distal e predispõe o desvio angular. ${ }^{4,29,31}$ o controle radiográfico em torno de 7 dias é fundamental para identificar possível perda de redução e viabilizar a reabordagem precoce. ${ }^{48}$ Nas primeiras 2 semanas, a manipulação com nova tentativa de redução fechada pode ser possível. $^{4}$

\section{Lesão neurológica}

O déficit neurológico é encontrado em cerca de 11\% das fraturas desviadas. ${ }^{41,49}$ A documentação do status sensitivo e motor préoperatório é fundamental: a presença do déficit indica maior gravidade e risco de lesão vascular associada, e ajuda a diferenciar a lesão traumática pré-operatória da iatrogênica. ${ }^{11,50}$

Historicamente, a lesão do nervo radial é descrita como a mais comum. ${ }^{1,14}$ No entanto, estudos mostram que a neuropraxia isolada do nervo interósseo anterior (NIA) é o tipo de lesão mais frequente na fratura em extensão, com incidência de cerca de 34\%. ${ }^{1,7,14,41}$ Por ser um ramo exclusivamente motor do nervo mediano, o diagnóstico é menos evidente. Em conjunto, as lesões do nervo mediano completas ou isoladas do NIA representam cerca de $60 \%$ das neuropraxias. ${ }^{49}$ Já na fratura em flexão, o nervo ulnar é o mais acometido, representando mais de $90 \%$ das neuropraxias. ${ }^{7,41}$

O prognóstico da lesão nervosa associada à FSU geralmente é bom, com recuperação completa na maioria dos pacientes. ${ }^{51} \mathrm{O}$ tempo médio para resolução do déficit é de pouco mais de 2 meses, com $60 \%$ dos casos apresentando melhora até o $3^{\circ}$ mês, e mais de $90 \%$ com restabelecimento total da função. ${ }^{1,49,52}$ Assim, a exploração cirúrgica não é recomendada rotineiramente nos casos de neuropraxia isolada. ${ }^{1,4,49}$

\section{Lesão vascular}

Ausência de pulso palpável na apresentação inicial é reportada entre 1 e $15 \%$ dos casos, chegando a $20 \%$ nas fraturas desviadas em algumas séries. ${ }^{1,4,8,22}$ Existe o risco de encarceramento do feixe neurovascular entre os fragmentos da fratura, lesão da íntima com formação de trombo tardio, laceração parcial, pseudoaneurisma ou até transecção total da artéria braquial e síndrome de compartimento. ${ }^{8}$ No entanto, pulso radial não palpável, apesar de indicar a urgência do tratamento, não significa necessariamente isquemia tecidual. Os vasos podem estar comprimidos pelo edema das partes moles adjacentes, apresentando espasmo ou mesmo encarcerados no foco da fratura, mas com um fluxo colateral adequado e perfusão distal suficiente. A reconstrução vascular raramente é necessária. ${ }^{4,8}$

Duas situações precisam ser diferenciadas: ausência de pulso radial palpável com a mão perfundida, rosada e quente; ausência de pulso radial com perfusão distal diminuída, mão pálida e fria. ${ }^{1,8,22}$ A primeira é uma urgência, requer atenção especial e prioridade no tratamento. A segunda é uma emergência que exige abordagem imediata. ${ }^{4}$ Em nenhuma situação deve-se aguardar o estudo vascular com angiografia ou doppler. A redução fechada com fixação percutânea é a primeira abordagem. ${ }^{8,9,50}$ Se continuar sem pulso após a redução anatômica e a estabilização da fratura, mas com a mão bem perfundida, rosada e quente, o paciente é mantido sob observação rigorosa até que o pulso seja palpável. ${ }^{22} \mathrm{~A}$ 
avaliação do fluxo arterial com doppler é indicada e a alta hospitalar adiada por pelo menos 24 horas. Deve-se realizar a exploração vascular se houver piora da perfusão nesse período. $^{8}$

Caso a mão se apresente com perfusão diminuída, pálida e fria após a redução fechada e fixação, é indicada a retirada dos pinos, redução abert,a e exploração vascular. ${ }^{4,9}$ Devido à possibilidade de espasmo arterial, é permitida tolerância de 10 a 15 minutos com o membro aquecido e o cotovelo estendido parcialmente antes de iniciar a exploração vascular. ${ }^{4} \mathrm{Na}$ ausência de reperfusão, a abordagem deve ser imediata. Nesse caso, é prudente solicitar a presença de cirurgião vascular ou microcirurgião para possível reconstrução arterial. ${ }^{8}$

O acesso transverso anterior é o recomendado, podendo ser ampliado para distal ou proximal e permitindo exploração direta do feixe neurovascular e reconstrução da artéria braquial quando necessário. ${ }^{1,4} \mathrm{Na}$ ausência de laceração ou transecção, o aquecimento do membro e aplicação de papaverina ou lidocaína tópica pode ajudar a diminuir o espasmo arterial. ${ }^{4}$ Monitoração rigorosa devido ao risco de síndrome de compartimento é mandatória. ${ }^{7}$ Apesar de não haver consenso sobre a indicação de fasciotomia descompressiva profilática do antebraço, esta deve ser considerada se o tempo de isquemia ultrapassar 6 horas. ${ }^{4}$

\section{Sindrome de compartimento}

A síndrome de compartimento, apesar de cada vez mais rara, com incidência em torno de 0.1 a $0.5 \%$, é a complicação mais devastadora da FSU. ${ }^{1,4,22}$ A melhoria da assistência em conjunto com maior atenção às técnicas de imobilização e abordagem cirúrgica das fraturas desviadas contribuem para a redução desta complicação; $;^{2,4}$ no entanto, o risco permanece. Alto índice de suspeição e abordagem precoce são mandatórios, podendo contribuir para resultados satisfatórios mesmo nos casos mais graves. A imobilização nunca deve ser com flexão acima de $80^{\circ}$, e o gesso cilíndrico deve ser evitado, dando-se preferência às talas gessadas posteriores bem acolchoadas. ${ }^{2}$ São considerados fatores de risco: crianças mais velhas, sexo masculino, fratura ipsilateral do antebraço (cotovelo flutuante), e lesão neurovascular. ${ }^{2,50} \mathrm{O}$ déficit do nervo mediano requer ainda mais atenção, pois a alteração da sensibilidade dolorosa pode mascarar 0 quadro. $^{1,4,14}$

\section{Rigidez articular}

A limitação da amplitude de movimento (ADM) no pósoperatório recente é comum; no entanto, a maioria dos pacientes evolui com melhora completa. ${ }^{27}$ Os fios de Kirschner devem ser retirados entre 3 e 4 semanas, tempo suficiente para a consolidação, evitando-se imobilização além desse período. Exercícios ativos são recomendados para ganho de ADM, e a fisioterapia raramente é indicada. ${ }^{53}$ Os principais fatores associados à rigidez articular são: lesão importante de partes moles, redução aberta (principalmente quando utilizado o acesso posterior), imobilização prolongada, e pacientes mais velhos. ${ }^{54,55}$

\section{Consolidação viciosa}

Lesão fisária (secundária ao trauma ou à cirurgia) é uma causa improvável de deformidade tardia. A consolidação viciosa é consequência da fratura mal-reduzida ou da falha de fixação. ${ }^{4}$ Em geral, a fratura com desvio póstero-medial gera deformidade em varo e fratura com desvio pósterolateral em valgo. 0 cúbito varo é descrito como complicação tardia mais frequente do que o cúbito valgo. ${ }^{15,56}$ Isso pode ser explicado não só pela maior incidência de fratura com desvio póstero-medial, mas também pelo fato de que a deformidade em varo é mais evidente, enquanto que um aumento do valgo pode ser negligenciado.

Um recurso que auxilia a prevenção da consolidação viciosa é a medida radiográfica do ângulo entre a linha fisária do capitelo e o eixo da diáfise, descrito por Baumann. ${ }^{4,57} \mathrm{~A}$ avaliação deve ser comparativa com o lado contra-lateral, mas, em geral, um valor acima de $80^{\circ}$ sugere alinhamento em varo. ${ }^{4}$ Os critérios descritos por Flynn ${ }^{58}$ para a avaliação do resultado tardio se baseiam na ADM do cotovelo (flexoextensão) e no ângulo de carregamento. $\mathrm{O}$ autor considerou o resultado insatisfatório quando encontrada uma variação maior que $15^{\circ}$ em relação ao lado contralateral. ${ }^{58}$

Em um estudo com seguimento médio de 6.6 anos, Moraleda et al. ${ }^{56}$ encontraram $36.9 \%$ de resultados insatisfatórios em 46 pacientes com fratura tipo II, que foram tratados de forma conservadora apenas com imobilização. Apesar de um bom resultado funcional ter sido encontrado na maioria dos casos, as fraturas tipo II não reduzidas evoluíram com deformidade em hiperextensão, limitação da flexão e cúbito varo em um número significativo de pacientes. ${ }^{56}$

Apesar de o cúbito varo ter sido considerado uma complicação principalmente estética, outras consequências da consolidação viciosa são descritas: maior risco de fratura do côndilo lateral, instabilidade rotatória póstero-lateral, dor, e paralisia tardia do nervo ulnar. ${ }^{4,7,59,60}$ A deformidade em varo desloca medialmente o eixo mecânico, o olécrano e o vetor de tração do tríceps. O torque em varo repetitivo no cotovelo leva ao estiramento crônico e consequente insuficiência do ligamento colateral lateral, resultando na instabilidade rotatória póstero-lateral. ${ }^{59} \mathrm{O}$ deslocamento da porção medial do tríceps durante a flexão do cotovelo traciona o nervo ulnar anteromedialmente e pode levar à neuropatia ulnar por atrito ou compressão dinâmica do tríceps contra o epicôndilo. ${ }^{4,60} \mathrm{~A}$ correção do cúbito varo na criança pode prevenir sequelas a longo prazo, e a crença de se tratar apenas de uma deformidade cosmética deve ser reconsiderada.

\section{Considerações finais}

É fundamental um cuidadoso exame físico na avaliação inicial, com atenção especial aos sinais de gravidade e fatores de risco para síndrome de compartimento. A ausência de pulso requer urgência e observação rigorosa, já a perfusão diminuída exige abordagem imediata. Deve-se buscar redução anatômica e um espaçamento máximo entre os pinos no foco da fratura para evitar a falha da fixação, sendo recomendado o uso de três pinos nas fraturas tipo III e IV. Conforme os conceitos apresentados, sugere-se um 


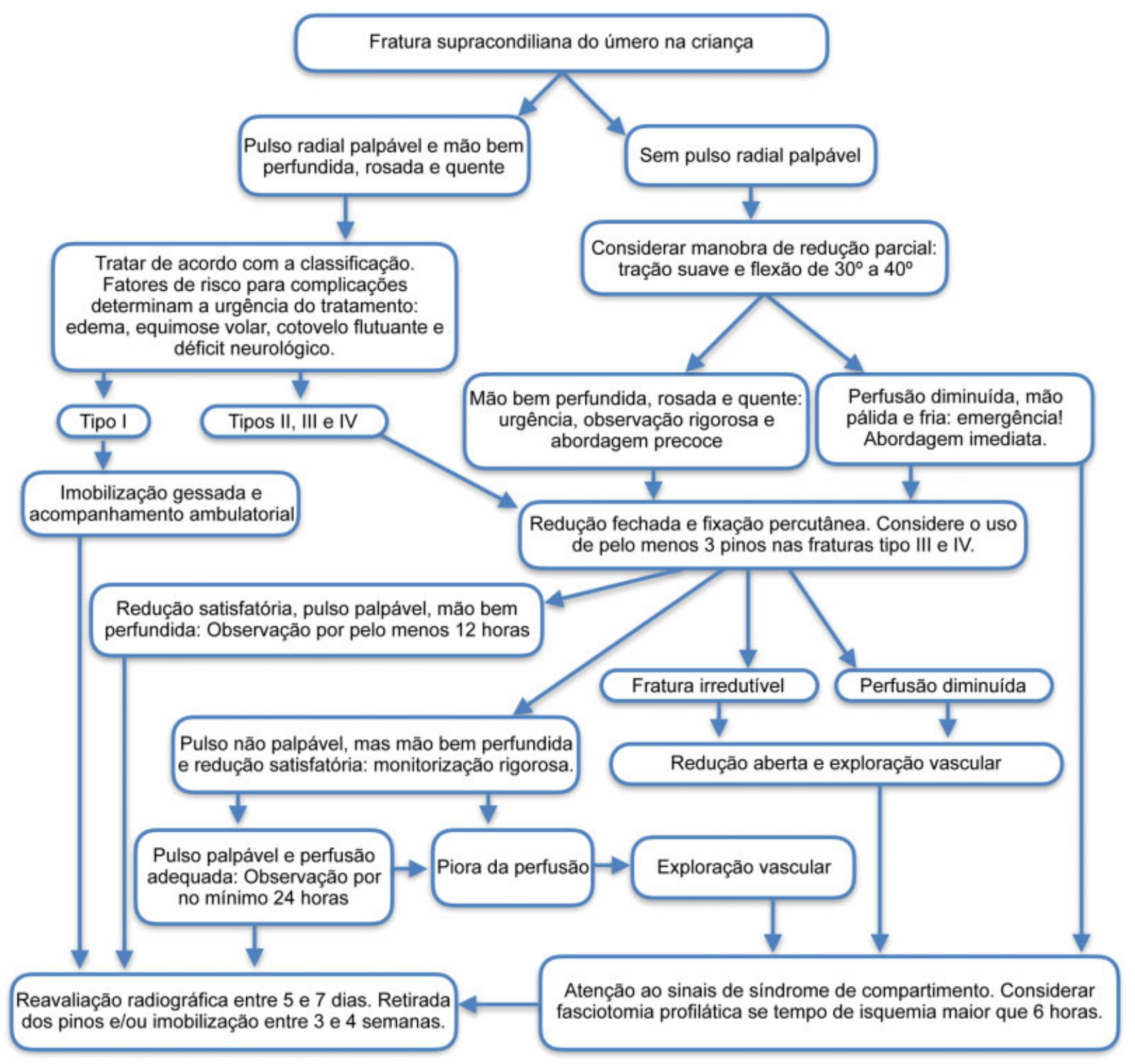

Fig. 5 Fluxograma para o tratamento da fratura supracondiliana do úmero na criança.

fluxograma para o tratamento da fratura supracondiliana do úmero na criança (-Figura 5).

Conflito de Interesses

Os autores declaram não haver conflito de interesses.

\section{Referências}

1 Omid R, Choi PD, Skaggs DL. Supracondylar humeral fractures in children. J Bone Joint Surg Am 2008;90(05):1121-1132

2 Robertson AK, Snow E, Browne TS, Brownell S, Inneh I, Hill JF. Who Gets Compartment Syndrome?: A Retrospective Analysis of the National and Local Incidence of Compartment Syndrome in Patients With Supracondylar Humerus Fractures J Pediatr Orthop 2018;38(05):e252-e256

3 Alton TB, Werner SE, Gee AO. Classifications in brief: the Gartland classification of supracondylar humerus fractures. Clin Orthop Relat Res 2015;473(02):738-741
4 Skaggs DL, Flynn JM. Supracondylar Fractures of the Distal Humerus. In: Waters PM, Skaggs DL, Flynn JM, Court-Brown CM, editors. Rockwood \& Wilkins' fractures in children. Philadelphia: Wolters Kluwer; 2010:754-844

5 Holt JB, Glass NA, Shah AS. Understanding the Epidemiology of Pediatric Supracondylar Humeral Fractures in the United States: Identifying Opportunities for Intervention. J Pediatr Orthop 2018; 38(05):e245-e251

6 Sankar WN, Hebela NM, Skaggs DL, Flynn JM. Loss of pin fixation in displaced supracondylar humeral fractures in children: causes and prevention. J Bone Joint Surg Am 2007;89(04):713-717

7 Abzug JM, Herman MJ. Management of supracondylar humerus fractures in children: current concepts. J Am Acad Orthop Surg 2012;20(02):69-77

8 Badkoobehi H, Choi PD, Bae DS, Skaggs DL. Management of the pulseless pediatric supracondylar humeral fracture. J Bone Joint Surg Am 2015;97(11):937-943

9 American Academy of Orthopaedic Surgeons. The Treatment of Pediatric Supracondylar Humerus Fractures. Rosemont, IL: AAOS; 2011 
10 American Academy of Orthopaedic Surgeons. Appropriate Use Criteria for the Management of Pediatric Supracondylar Humerus Fractures. Rosemont, IL: AAOS; 2014

11 Ho CA, Podeszwa DA, Riccio AI, Wimberly RL, Ramo BA. Soft Tissue Injury Severity is Associated With Neurovascular Injury in Pediatric Supracondylar Humerus Fractures. J Pediatr Orthop 2018;38 (09):443-449

12 Flynn K, Shah AS, Brusalis CM, Leddy K, Flynn JM. Flexion-Type Supracondylar Humeral Fractures: Ulnar Nerve Injury Increases Risk of Open Reduction. J Bone Joint Surg Am 2017;99(17):1485-1487

13 Gartland JJ. Management of supracondylar fractures of the humerus in children. Surg Gynecol Obstet 1959;109(02):145-154

14 Wilkins KE. Supracondylar fractures: what's new? J Pediatr Orthop B 1997;6(02):110-116

15 Spencer HT, Dorey FJ, Zionts LE, et al. Type II supracondylar humerus fractures: can some be treated nonoperatively? J Pediatr Orthop 2012;32(07):675-681

16 Ariyawatkul T, Eamsobhana P, Kaewpornsawan K. The necessity of fixation in Gartland type 2 supracondylar fracture of the distal humerus in children (modified Gartland type 2A and 2B). J Pediatr Orthop B 2016;25(02):159-164

17 Silva M, Delfosse EM, Park H, Panchal H, Ebramzadeh E. Is the "Appropriate Use Criteria" for Type II Supracondylar Humerus Fractures Really Appropriate? J Pediatr Orthop 2019;39(01):1-7

18 Leitch KK, Kay RM, Femino JD, Tolo VT, Storer SK, Skaggs DL. Treatment of multidirectionally unstable supracondylar humeral fractures in children. A modified Gartland type-IV fracture. J Bone Joint Surg Am 2006;88(05):980-985

19 Novais EN, Andrade MAP, Gomes DC. The use of a joystick technique facilitates closed reduction and percutaneous fixation of multidirectionally unstable supracondylar humeral fractures in children. J Pediatr Orthop 2013;33(01):14-19

20 Mulpuri K, Wilkins K. The treatment of displaced supracondylar humerus fractures: evidence-based guideline. J Pediatr Orthop 2012;32(02, Suppl 2):S143-S152

21 Garg S, Weller A, Larson AN, et al. Clinical characteristics of severe supracondylar humerus fractures in children. J Pediatr Orthop 2014;34(01):34-39

22 Choi PD, Melikian R, Skaggs DL. Risk factors for vascular repair and compartment syndrome in the pulseless supracondylar humerus fracture in children. J Pediatr Orthop 2010;30(01):50-56

23 Mehlman CT, Strub WM, Roy DR, Wall EJ, Crawford AH. The effect of surgical timing on the perioperative complications of treatment of supracondylar humeral fractures in children. J Bone Joint Surg Am 2001;83(03):323-327

24 Gupta N, Kay RM, Leitch K, Femino JD, Tolo VT, Skaggs DL. Effect of surgical delay on perioperative complications and need for open reduction in supracondylar humerus fractures in children. J Pediatr Orthop 2004;24(03):245-248

25 Abbott MD, Buchler L, Loder RT, Caltoum CB. Gartland type III supracondylar humerus fractures: outcome and complications as related to operative timing and pin configuration. J Child Orthop 2014;8(06):473-477

26 Bales JG, Spencer HT, Wong MA, Fong YJ, Zionts LE, Silva M. The effects of surgical delay on the outcome of pediatric supracondylar humeral fractures. J Pediatr Orthop 2010;30(08):785-791

27 Skaggs DL, Cluck MW, Mostofi A, Flynn JM, Kay RM. Lateral-entry pin fixation in the management of supracondylar fractures in children. J Bone Joint Surg Am 2004;86(04):702-707

28 Pennock AT, Charles M, Moor M, Bastrom TP, Newton PO. Potential causes of loss of reduction in supracondylar humerus fractures. J Pediatr Orthop 2014;34(07):691-697

29 Bloom T, Robertson C, Mahar AT, Newton P. Biomechanical analysis of supracondylar humerus fracture pinning for slightly malreduced fractures. J Pediatr Orthop 2008;28(07):766-772

30 Wallace M, Johnson DB Jr, Pierce W, Iobst C, Riccio A, Wimberly RL. Biomechanical Assessment of Torsional Stiffness in a Supracon- dylar Humerus Fracture Model. J Pediatr Orthop 2019;39(03): e210-e215

31 Bauer JM, Stutz CM, Schoenecker JG, Lovejoy SA, Mencio GA, Martus JE. Internal Rotation Stress Testing Improves Radiographic Outcomes of Type 3 Supracondylar Humerus Fractures. J Pediatr Orthop 2019;39(01):8-13

32 Zenios M, Ramachandran M, Milne B, Little D, Smith N. Intraoperative stability testing of lateral-entry pin fixation of pediatric supracondylar humeral fractures. J Pediatr Orthop 2007;27(06): 695-702

33 Gottschalk HP, Sagoo D, Glaser D, Doan J, Edmonds EW, Schlechter J. Biomechanical analysis of pin placement for pediatric supracondylar humerus fractures: does starting point, pin size, and number matter? J Pediatr Orthop 2012;32(05):445-451

34 Kocher MS, Kasser JR, Waters PM, et al. Lateral entry compared with medial and lateral entry pin fixation for completely displaced supracondylar humeral fractures in children. A randomized clinical trial. J Bone Joint Surg Am 2007;89(04):706-712

35 Lee KM, Chung CY, Gwon DK, et al. Medial and lateral crossed pinning versus lateral pinning for supracondylar fractures of the humerus in children: decision analysis. J Pediatr Orthop 2012;32 (02):131-138

36 Gaston RG, Cates TB, Devito D, et al. Medial and lateral pin versus lateral-entry pin fixation for Type 3 supracondylar fractures in children: a prospective, surgeon-randomized study. J Pediatr Orthop 2010;30(08):799-806

37 Lee YH, Lee SK, Kim BS, et al. Three lateral divergent or parallel pin fixations for the treatment of displaced supracondylar humerus fractures in children. J Pediatr Orthop 2008;28(04):417-422

38 Skaggs DL, Hale JM, Bassett J, Kaminsky C, Kay RM, Tolo VT. Operative treatment of supracondylar fractures of the humerus in children. The consequences of pin placement. J Bone Joint Surg Am 2001;83(05):735-740

39 Silva M, Knutsen AR, Kalma JJ, et al. Biomechanical testing of pin configurations in supracondylar humeral fractures: the effect of medial column comminution. J Orthop Trauma 2013;27(05):275-280

40 Woratanarat P, Angsanuntsukh C, Rattanasiri S, Attia J, Woratanarat T, Thakkinstian A. Meta-analysis of pinning in supracondylar fracture of the humerus in children. J Orthop Trauma 2012;26 (01):48-53

41 Babal JC, Mehlman CT, Klein G. Nerve injuries associated with pediatric supracondylar humeral fractures: a meta-analysis. J Pediatr Orthop 2010;30(03):253-263

42 Slobogean BL, Jackman H, Tennant S, Slobogean GP, Mulpuri K. Iatrogenic ulnar nerve injury after the surgical treatment of displaced supracondylar fractures of the humerus: number needed to harm, a systematic review. J Pediatr Orthop 2010;30(05): 430-436

43 Silva M, Cooper SD, Cha A. The Outcome of Surgical Treatment of Multidirectionally Unstable (Type IV) Pediatric Supracondylar Humerus Fractures. J Pediatr Orthop 2015;35(06):600-605

44 Kwak-Lee J, Kim R, Ebramzadeh E, Silva M. Is medial pin use safe for treating pediatric supracondylar humerus fractures? J Orthop Trauma 2014;28(04):216-221

45 Ay S, Akinci M, Kamiloglu S, Ercetin O. Open reduction of displaced pediatric supracondylar humeral fractures through the anterior cubital approach. J Pediatr Orthop 2005;25(02):149-153

46 Mahan ST, May CD, Kocher MS. Operative management of displaced flexion supracondylar humerus fractures in children. J Pediatr Orthop 2007;27(05):551-556

47 Novais EN, Carry PM, Mark BJ, De S, Miller NH. Posterolaterally displaced and flexion-type supracondylar fractures are associated with a higher risk of open reduction. J Pediatr Orthop B 2016; 25(05):406-411

48 Karalius VP, Stanfield J, Ashley P, et al. The Utility of Routine Postoperative Radiographs After Pinning of Pediatric Supracondylar Humerus Fractures. J Pediatr Orthop 2017;37(05):e309-e312 
49 Shore BJ, Gillespie BT, Miller PE, Bae DS, Waters PM. Recovery of Motor Nerve Injuries Associated With Displaced, Extension-type Pediatric Supracondylar Humerus Fractures. J Pediatr Orthop 2019;39(09):e652-e656

50 Harris LR, Arkader A, Broom A, et al. Pulseless Supracondylar Humerus Fracture With Anterior Interosseous Nerve or Median Nerve Injury-An Absolute Indication for Open Reduction? J Pediatr Orthop 2019;39(01):e1-e7

51 Valencia M, Moraleda L, Díez-Sebastián J. Long-term Functional Results of Neurological Complications of Pediatric Humeral Supracondylar Fractures. J Pediatr Orthop 2015;35(06):606-610

52 Patriota GS, Assunção Filho CA, Assunção CA. Qual a Melhor Técnica para Fixação no Tratamento de Fratura Supracondilar do Úmero em Crianças? Rev Bras Ortop 2017;52(04):428-434

53 Schmale GA, Mazor S, Mercer LD, Bompadre V. Lack of Benefit of Physical Therapy on Function Following Supracondylar Humeral Fracture: A Randomized Controlled Trial. J Bone Joint Surg Am 2014;96(11):944-950

54 Spencer HT, Wong M, Fong YJ, Penman A, Silva M. Prospective longitudinal evaluation of elbow motion following pediatric supracondylar humeral fractures. J Bone Joint Surg Am 2010;92(04):904-910
55 Fletcher ND, Schiller JR, Garg S, et al. Increased severity of type III supracondylar humerus fractures in the preteen population. J Pediatr Orthop 2012;32(06):567-572

56 Moraleda L, Valencia M, Barco R, González-Moran G. Natural history of unreduced Gartland type-II supracondylar fractures of the humerus in children: a two to thirteen-year follow-up study. J Bone Joint Surg Am 2013;95(01):28-34

57 Worlock P. Supracondylar fractures of the humerus. Assessment of cubitus varus by the Baumann angle. J Bone Joint Surg $\operatorname{Br} 1986$; 68(05):755-757

58 Flynn JC, Matthews JG, Benoit RL. Blind pinning of displaced supracondylar fractures of the humerus in children. Sixteen years' experience with long-term follow-up. J Bone Joint Surg Am 1974; 56(02):263-272

59 O'Driscoll SW, Spinner RJ, McKee MD, et al. Tardy posterolateral rotatory instability of the elbow due to cubitus varus. J Bone Joint Surg Am 2001;83(09):1358-1369

60 Spinner RJ, O'Driscoll SW, Davids JR, Goldner RD. Cubitus varus associated with dislocation of both the medial portion of the triceps and the ulnar nerve. J Hand Surg Am 1999;24(04): $718-726$ 